Draft VERSion June 9, 2021

Preprint typeset using $\mathrm{LATE}_{\mathrm{E}} \mathrm{X}$ style emulateapj v. 5/2/11

\title{
MULTI-WAVELENGTH OBSERVATIONS OF COMET C/2011 L4 (PAN-STARRS)
}

\author{
Bin Yang ${ }^{1,2}$, Jacqueline Keane ${ }^{1}$, Karen Meech ${ }^{1,3}$, Tobias Owen ${ }^{3}$, Richard Wainscoat ${ }^{3}$ \\ 1 NASA Astrobiology Institute, University of Hawaii, Honolulu, HI 96822 \\ 2 European Southern Observatory, Santiago, Chile \\ 3 Institute for Astronomy, University of Hawaii, Honolulu, HI 96822
}

Draft version June 9, 2021

\begin{abstract}
Dynamically new comet C/2011 L4 (PanSTARRS) is one of the brightest comets since the great comet C/1995 O1 (Hale-Bopp). Here, we present our multi-wavelength observations of C/2011 L4 during its in-bound passage to the inner Solar system. A strong absorption band of water ice at $2.0 \mu \mathrm{m}$ was detected in the near infrared spectra, taken with the 8-m Gemini-North and 3-m IRTF telescopes. The companion $1.5 \mu \mathrm{m}$ band of water ice, however, was not observed. Spectral modeling show that the absence of the $1.5 \mu \mathrm{m}$ feature can be explained by the presence of sub-micron-sized fine ice grains. No gas lines (i.e. $\mathrm{CN}, \mathrm{HCN}$ or $\mathrm{CO}$ ) were observed pre-perihelion either in the optical or in the sub-millimeter. 3- $\sigma$ upper limits to the $\mathrm{CN}$ and $\mathrm{CO}$ production rates were derived. The comet exhibited a very strong continuum in the optical and its slope seemed to become redder as the comet approached the Sun. Our observations suggest that C/2011 L4 is an unusually dust-rich comet with a dust-to-gas mass ratio $>4$.
\end{abstract}

Subject headings: comets: individual (C/2011 L4) —infrared: planetary systems - Oort Cloud

\section{INTRODUCTION}

Comet C/2011 L4 (PanSTARRS) was discovered at 7.9 AU from the Sun in June 2011 by the 1.8-m PanSTARRS 1 survey telescope atop Haleakala. The detection was confirmed in followup observations with the Canada-France-Hawaii Telescope (Wainscoat et al. 2011). The small reciprocal of the original semi-major axis, $1 / a=-8.9 \times 10^{-5} \mathrm{AU}^{-1}$ (Williams 2013), suggesting that this comet is a recent arrival from the Oort Cloud. C/2011 L4 reached an apparent visual magnitude of -1 at $0.301 \mathrm{AU}$ in March 2013, which made it one of the brightest comets in the past two decades since comet C/1995 O1 (Hale-Bopp). The apparition of C/2011 L4 provided a rare opportunity to monitor a dynamically new comet over a significant time period and a wide range of heliocentric distances, particularly during the in-bound leg.

At the time of discovery, C/2011 L4 showed an extended appearance (Wainscoat et al. 2011), indicating the presence of a substantial coma. The turn-on distance for any appreciable water ice sublimation is around 5-6 AU from the Sun, beyond which the equilibrium surface temperatures is too low and $\mathrm{H}_{2} \mathrm{O}$-ice sublimation is not sufficient to lift even the smallest sub-micron-sized dust particles from the surface (Meech \& Svoren 2004). It is clear that $\mathrm{C} / 2011 \mathrm{~L} 4$ was far beyond the water sublimating zone yet it appeared active. Several possible mechanisms that could power distant comet activity have been proposed, including latent heat release from the amorphous-to-crystalline water ice transition (Prialnik 1992; Notesco et al. 2003; Bar-Nun \& Laufer 2003), sublimation of frozen super-volatiles (Meech \& Svoren 2004) and comet fragmentation (Boehnhardt 2004). Among these, the exothermic amorphous-to-crystalline phase transition of water ice is considered to be an important energy source that can trigger distant cometary

yangbin@ifa.hawaii.edu activity and power a cometary outburst Enzian et al. 1997). Observationally, crystalline ice differs from its amorphous counterpart by the presence of a sharp narrow feature at $1.65 \mu \mathrm{m}$. This feature has been observed in large Kuiper belt objects via near infrared (NIR) spectroscopy Jewitt \& Luu 2004; Trujillo et al. 2007) and was recently detected in the coma of an outbursting Jupiter-family comet (JFC) P/2010 H2 (Vales) (Yang \& Sarid 2010). However, crystalline water ice has never been seen in any Oort Cloud comet (OCC) (Davies et al. 1997; Kawakita et al. 2004). Nevertheless, the absence of observational evidences does not exclude the presence of crystalline ice in OCCs. This is because the available OCC spectra have limited signal-to-noise ratios (SNR) and spectral resolutions. We conducted a multi-wavelength observational campaign of C/2011 L4, from optical to sub-millimeter wavelengths. The main goals of this study were three-fold: (i) search for water ice, in particular, the crystalline feature at $1.65 \mu \mathrm{m}$ via high SNR and medium-resolution spectroscopy in the NIR; (ii) monitor the on-set of volatile species (such as $\mathrm{CN}, \mathrm{HCN}$ and $\mathrm{CO}$, etc) in the optical and the radio as the comet approached the Sun, (iii) monitor the development of the dust and gas comae via optical spectroscopy.

\section{OBSERVATIONS AND DATA REDUCTIONS}

NIR spectroscopy was conducted using the GNIRS spectrograph on the 8-m Gemini North (Gemini-N) telescope and the SpeX spectrograph on the 3-m IRTF telescope. The GNIRS observations were taken with the short camera (plate scale: $0.15^{\prime \prime} /$ pix). The SXD cross-dispersed mode, $32 \mathrm{l} / \mathrm{mm}$ grating and 1.0" slit, provides an averaged resolving power of $\mathrm{R} \sim 500$ over 0.85-2.5 $\mu \mathrm{m}$. The SpeX observations were made using the high throughput prism mode $(0.8-2.5 \mu \mathrm{m})$ and a $0.8^{\prime \prime} \times 15^{\prime \prime}$ slit that provides a spectral resolution of $R \sim$ 150 (Rayner et al. 2003). Two nearby G-type stars, used 
both as the telluric correction standard stars and solar analogs, were observed together with the comet during each run. The GNIRS data were reduced using the Image Reduction and Analysis Facility (IRAF) software and the Gemini IRAF package. The SpeX data were reduced using the reduction pipeline SpeXtool Cushing et al. 2004).

Optical spectra were obtained using the Gemini MultiObject Spectrograph (GMOS) spectrograph on Gemini$\mathrm{N}$ with the long-slit mode (plate scale: $0.146^{\prime \prime} / \mathrm{pix}$ ). We used the B600 grating and set the slit width to $1.5^{\prime \prime}$, which provides a resolving power of $\mathrm{R} \sim 560$. At least one G-type star was observed together with the comet during each observing run. In addition, one spectrophotometric star was observed for flux calibration. The GMOS data were reduced using a combination of the Gemini IRAF package and the XIDL code (J. X. Prochaska, priv. communication).

Besides, we also obtained submillimeter spectroscopy of C/2011 L4 using the 15-m JCMT Telescope on Mauna Kea, Hawaii. The ACSIS spectrometer was used, providing a total bandwidth of $250 \mathrm{MHz}$ and spectral channel spacing of $30.5 \mathrm{kHz}$. The comet was observed over three separate observing runs between August and November 2012. All of our observations were performed with the HARP receiver in position-switching mode $\left(5^{\prime}\right.$ apart in azimuth). The data were reduced using a combination of Starlink and IDL software. A record of the observations is provided in Table 1 .

\section{RESULTS}

\subsection{Detection of Water Ice}

The NIR observations of C/2011 L4 are presented in Figure 1. A strong absorption feature centered at $2.0 \mu \mathrm{m}$ was consistently observed both in the SpeX and the GNIRS spectra. The profile and the center of this absorption feature is consistent with the diagnostic 2.0$\mu \mathrm{m}$ absorption band of water ice. However, water ice, if present, usually exhibits two absorption bands simultaneously, i.e. the $2.0 \mu \mathrm{m}$ band and an accompanying band at $1.5 \mu \mathrm{m}$. The spectra obtained in March and June appear featureless between 1.4 and $1.7 \mu \mathrm{m}$. The July spectrum, however, shows two small absorption features at 1.28 and $1.50 \mu \mathrm{m}$, respectively. The latter feature could be associated with water ice but it is significantly narrower than the $1.5 \mu \mathrm{m}$ ice band. Comparing the comet spectrum with the standard star spectra taken in July, we conclude that these two minor features are more likely due to incomplete cancellations of telluric absorptions. The apparent absence of the $1.5 \mu \mathrm{m}$ feature in contrast to the presence of the strong $2.0 \mu \mathrm{m}$ band is not unique to $\mathrm{C} / 2011 \mathrm{~L} 4$. For instance, the outbursting comet $17 \mathrm{P} /$ Holmes exhibited strong absorption bands at 2.0 and $3.0 \mu \mathrm{m}$ without showing any sign of the $1.5 \mu \mathrm{m}$ band (Yang et al. 2009). Attempting to understand the problem of the missing $1.5 \mu \mathrm{m}$ feature, Yang et al. (2009) investigated the effects of impurity and particle size on the synthesized ice spectra. They concluded that neither of the investigated properties could explain the asymmetry between the 1.5 and $2.0 \mu \mathrm{m}$ bands. However, in the study of $17 \mathrm{P}$, the adopted Hapke theory has significant limitations and cannot account for particles smaller than the wavelength being studied (Hapke 1993). In this paper, we apply Mie theory to synthesize water ice spectra and the optical constants from Mastrapa et al. (2009). The main reason for choosing Mie over Hapke is that Mie theory is suitable for investigating small particles that have Rayleigh scattering parameter $X=2 \pi a_{g} / \lambda$ much less than one, where $a_{g}$ is particle radius and $\lambda$ is the wavelength.

Using the new Mie model, we found that the ratio between the depth of the 1.5 and $2.0 \mu \mathrm{m}$ bands is sensitive to grain size. Using sub-micron fine grains, our best-fit areal mixing models can successfully reproduce the observed spectral features, i.e. a strong $2.0 \mu \mathrm{m}$ band and a much weaker $1.5 \mu \mathrm{m}$ band. All the comet spectra can be explained by roughly $30 \%$ fine-grained water ice $\left(a_{g} \sim 0.2 \mu \mathrm{m}\right)$ mixed with spectrally featureless materials (such as amorphous carbon). We note that the strength of the $2.0 \mu \mathrm{m}$ band remained consistent from March to July, indicating that the dust-to-ice ratio of the coma did not change significantly over heliocentric distances from 5.24 to 3.68 AU. The persistence of the ice features suggests that the lifetime of the ice particles must be longer or comparable to the slit-crossing time. For the July run, the extraction aperture radius is $2.5^{\prime \prime}$ (or $\sim 6700 \mathrm{~km}$ projected in the sky). Assuming the expansion speed of the icy grains is represented by the empirical relation: $v_{d u s t}=0.535 \cdot r_{h}^{-0.6} \mathrm{~km} / \mathrm{s}$ (Bobrovnikoff 1954; Whipple 1978), the slit-crossing time is at least 7.6 hours. As shown in Lien (1990) and Beer et al. (2006), the lifetime of a sub-micron dirty ice grain at $3.68 \mathrm{AU}$ is less than 2 hours. If dirty icy particles were to survive the slit-crossing, their outflow velocity would have to exceed $1 \mathrm{~km} / \mathrm{s}$, which is non-physical for the comet at $r_{h}>3.5 \mathrm{AU}$. Given the estimates above, the ice particles detected in comet $\mathrm{C} / 2011$ L4 it is likely to be pure.

\subsection{Optical Spectroscopy}

Optical spectroscopy was obtained using Gemini-N in queue mode from April to July 2012. Specifically, we searched for the $\mathrm{CN} 3880 \AA$ emission band as a probe to monitor the sublimation of volatile species. After subtracting the scaled solar analog continua, the residual spectra appear featureless with no apparent cometary emission lines detected (see Figure 3a). Upper limits to the $\mathrm{CN}$ production rate were quantified using the standard errors in the two wavelength regions, 3900-3950^ and $3950-4010 \AA$, of the residual spectra. Details of our method are described in Hsieh et al. (2012). 3- $\sigma$ limits to the $\mathrm{CN}$ band flux and the $\mathrm{CN}$ production rate are listed in Table 2. The derived upper limits $(5.0-6.0 \times 10$ $27 \mathrm{~mole} / \mathrm{s}$ ) to the water production rate of $\mathrm{C} / 2011 \mathrm{~L} 4$, are consistent with the $\mathrm{H}_{2} \mathrm{O}$ production rate of $5.1 \times 10^{27}$ $\mathrm{mol} / \mathrm{s}$ in September 2012, reported by Biver et al. (2012).

Since no gas emission was detected, the continuum must be solely controlled by the dust coma. To assess the strength of the dust continuum, we calculate Af $\rho$ (A'Hearn et al. 1984) and list our results in Table 2. The Af $\rho$ of $\mathrm{C} / 2011$ L4 between 4900 and $5500 \AA$ exhibits small but steady increases from April to July, which are consistent with an independent photometric study of this comet (Ivanova et al. 2014), obtained during a similar period of time. In terms of Af $\rho, \mathrm{C} / 2011$ L4 dwarfs the majority of observed OCCs (Storrs et al. 1992; Langland-Shula \& Smith 2011). However, it is 
an order of magnitude lower than that of C/1995 O1 (Weiler et al. 2003). We further estimated the dust mass loss rate, using the formulations in Weiler et al. (2003) and Agarwal et al. (2007). Given that the gas production rate was low and our NIR observations suggest the presence of sub-micron ice particles, we assumed a differential size distribution of $\mathrm{f}(\mathrm{a}) \sim \mathrm{a}^{-4}$, which gives an effective mean dust size: $\bar{a}=\frac{\int \pi a^{3} f(a)}{\int \pi a^{2} f(a)}<1 \mu \mathrm{m}$. For dust velocity, we adopted an outflow velocity relationship of $v_{\text {dust }}(a)=0.5^{*} \sqrt{\frac{C \cdot Q_{s}}{\rho_{d} \cdot a}} \mathrm{~km} / \mathrm{s}$ from Lisse et al. (1998), where $\mathrm{C}=0.595 \times 10^{-3} \mathrm{~kg} \mathrm{~m}^{-2}$, the scattering efficiency $Q_{s} \sim 1$ and the dust bulk density $\rho_{d}=10^{3} \mathrm{~kg} \mathrm{~m}^{-3}$. Integrating over a size interval from $0.1 \mu \mathrm{m}$ to $1 \mathrm{~cm}$ and assuming a geometric albedo of 0.04 for dust particles (Singh et al. 1992), we compute the dust mass-loss-rate of C/2011 L4 to be about $700 \mathrm{~kg} / \mathrm{s}$. Using the upper limit to the $\mathrm{CN}$ production, we estimated that the upper limit to the gas mass-loss-rate is $\sim 200 \mathrm{~kg} / \mathrm{s}$. Shown in Table 2 , the dust-to-gas mass ratio $(\chi)$ of C/2011 L4 is > 4 in July 2012. In comparison, $\mathrm{C} / 1995 \mathrm{O} 1$ holds the highest $\chi$ that is $\gtrsim 5$ (Jewitt \& Matthews 1999) while most comets have $\chi$ less than 2 (Singh et al. 1992; Sanzovo et al. 1996). Our results indicate that C/2011 L4 is an unusually dustrich comet. We note that the dust size distribution and the dust outflow velocity are the most uncertain factors in calculating the dust production rate. If we assume $\mathrm{f}(\mathrm{a}) \sim \mathrm{a}^{-3.5}$, the corresponding dust-to-gas ratio would be about 100 , which is highly unlikely. Detailed models that fully explore the parameter space for these two factors are beyond the scope of this paper.

\subsection{Submillimeter Observation}

In the radio, $\mathrm{CO}$ and $\mathrm{HCN}$ are the most easily detected gaseous species for distant comets ( $\mathrm{r}>3 \mathrm{AU}$ ) (Senay \& Jewitt 1994; Biver et al. 2002). We carried out three observing runs in the sub-millimeter to search for $\mathrm{CO}$ and $\mathrm{HCN}$. No gas emission was detected from August to November. To quantify the upper limits to the production rates of $\mathrm{CO}$ and $\mathrm{HCN}$, we calculated the standard errors in two background regions and integrated line intensities $\left(f_{C O}\right.$ and $\left.f_{H C N}\right)$ within a $1.2 \mathrm{~km} \mathrm{~s}^{-1}$ band. Even at the smallest $r(2.3 \mathrm{AU})$, where the $\mathrm{CO}$ lifetime is the shortest, the expected lifetime against photo-destruction for a CO molecule is $\tau_{C O}=3.6 \times 10^{6} \cdot r_{h}^{2}=1.9 \times 10^{7} \mathrm{sec}$ (about 1 year). Similarly, $\tau_{H C N}=4.8 \times 10^{5} \mathrm{sec}$ (about 4 days). Therefore, we ignored photo-destruction effect for both $\mathrm{CO}$ and HCN. We assumed gas molecules escaping from the surface at a constant velocity and adopted an average gas expansion velocity of $0.8 \cdot \mathrm{r}^{-0.5} \mathrm{~km} \mathrm{~s}^{-1}$ (Biver et al., 1999, 2000). 3- $\sigma$ upper limits to the production rates were computed for two kinetic temperatures, i.e $\mathrm{T}=10 \mathrm{~K}$ (higher values) and $\mathrm{T}=50 \mathrm{~K}$ (lower values). For August, $f_{C O}=0.015[\mathrm{~K} \mathrm{~km} / \mathrm{s}], \mathrm{Q}_{C O}<1.5-4.6 \times 10^{27}$, $f_{H C N}=0.032[\mathrm{~K} \mathrm{~km} / \mathrm{s}], Q_{H C N}<0.5-3.1 \times 10^{25}$; for October, $f_{C O}=0.024[\mathrm{~K} \mathrm{~km} / \mathrm{s}], \mathrm{Q}_{C O}<2.4-7.4 \times 10^{27}, f_{H C N}=$ $0.035[\mathrm{~K} \mathrm{~km} / \mathrm{s}], \mathrm{Q}_{H C N}<0.5-3.3 \times 10^{25}$; and for November, $f_{C O}=0.093[\mathrm{~K} \mathrm{~km} / \mathrm{s}], \mathrm{Q}_{C O}<0.9-2.8 \times 10^{28}, f_{H C N}=$ $0.037[\mathrm{~K} \mathrm{~km} / \mathrm{s}], \mathrm{Q}_{H C N}<0.6-3.6 \times 10^{25}$. The much higher upper limit on $\mathrm{Q}_{C O}$ derived for the November run is due to poor weather conditions.

\section{DISCUSSION}

\subsection{Water Ice Features}

The critical temperature for amorphous-to-crystalline ice transition is $\mathrm{T}_{c} \sim 140 \mathrm{~K}$, at which the timescale of crystallization is about 1 hour. The highest possible surface temperature for C/2011L4 should be that at its subsolar point, where $\mathrm{T}_{s s}\left(\mathrm{r}_{h}\right)=\mathrm{T}_{0} / \sqrt{r_{h}}$. If we assume $\mathrm{C} / 2011 \mathrm{~L} 4$ is a slow rotator, then $\mathrm{T}_{0}=392 \mathrm{~K}$ (Jewitt 2009). At $\mathrm{r}_{h}=5.2 \mathrm{AU}, \mathrm{C} / 2011 \mathrm{~L} 4$ can reach $\mathrm{T}_{s s} \sim 170 \mathrm{~K}$ by solar heating alone. Although this is an extreme case, it shows that it is possible that at least part of the surface or near-surface ice could have experienced crystallization within our observation window.

As shown in Figure 2, sub-micron-sized ice grains show a very weak or non-existent absorbing feature at $1.65 \mu \mathrm{m}$ regardless of the physical structure of individual grains. Thus, the absence of the $1.65 \mu \mathrm{m}$ band does not exclude the presence of very fine crystalline ice grains. When focusing on the $2.0 \mu \mathrm{m}$ band, our $\chi^{2}$-fit show that amorphous ice fits the comet spectra better than crystalline ice for the March and June data, while the crystalline model fits the July data marginally better. However, the small difference between these two ice models is within the noise level of our data. Therefore, we cannot constrain the crystallinity of the icy grains of C/2011 L4, mainly due to their small sizes.

Although the observed asymmetry in absorption strength between the bands at $1.5 \mu \mathrm{m}$ and $2.0 \mu \mathrm{m}$ can be successfully explained by sub-micron pure water ice grains, there are other explanations for the observed features. For example, the $2.0 \mu \mathrm{m}$ absorption feature may not be solely generated by pure water ice grains but in addition be due to the superposition another absorption band of organic materials or hydrated minerals. We explored this possibility by searching for matching materials that exhibit a prominent absorption feature at or near $2.0 \mu \mathrm{m}$. We investigated several commonly used spectral libraries, such as RELAB (Hiroi et al. 1996), USGS (Clark et al. 2003) and ASTER (Baldridge et al. 2009), which include examined materials from terrestrial minerals to meteorite samples. We find no other material with a NIR signature that matches the shape and position of the $2.0 \mu \mathrm{m}$ band of $\mathrm{C} / 2011 \mathrm{~L} 4$.

\subsection{Dust Coma}

Shown in Fig 3b, the slope of the dust continuum became steeper as the comet approached the Sun. Similar color- $r_{h}$ trends have been reported in other comets (Hartmann \& Cruikshank 1984). However, later studies (Jewitt \& Meech 1988) did not confirm these reports, suggesting instead that the reported trend may have been due to the contamination of cometary emissions. Given that no cometary emissions were observed in our optical data, the observed color trend is not caused by gas contamination. Possible explanations for this correlation, if real, include: (a) increase in the effective size of coma grains, (b) decrease in the ice-to-dust ratio in coma grains and (c) changes in phase angle as the comet approaches the Sun. Our NIR observations suggest that the ice-to-dust ratio remained more or less the same (within uncertainties) and the phase angle of C/2011 L4 did not change much from April to July. Therefore both (b) and (c) scenarios are unlikely and we are left with (a) as the only plausible mechanism: the observed color- $r_{h}$ trend 
is observed because larger dust particles being released into the coma as the comet moved closer to the Sun.

Given that no gas was detected, what could be the driving force for the cometary activity? One possibility is that C/2011 L4 underwent a strong outburst which released most of the observed dust and ice grains into the coma. At the time of our observations, the major gas production had ceased and the nucleus was surrounded by a remnant dust cloud. However, the change in the slope of the dust continuum argues for an on-going mass loss process. C/2011 L4 is a dynamically new comets, so reactive species could have been synthesized via long term exposure to cosmic rays or galactic UV bombardment while the comet was in the Oort Cloud (Hughes 1990). These radical species could explode when exposed to the solar wind and that could in turn trigger several small outbursts (Niedner 1980). Alternatively, the distant activity of $\mathrm{C} / 2011$ L4 could be driven by highly volatile ices, such as $\mathrm{CO}_{2}$. Recently, several comets, such as comet 103P/Hartley 2 and comet ISON, haven observed to show strong $\mathrm{CO}_{2}$ signatures A'Hearn et al.
2011; Lisse et al. 2013). Unfortunately, $\mathrm{CO}_{2}$ can not be observed from the ground and no space observations are available for $\mathrm{C} / 2011 \mathrm{~L} 4$. To verify whether $\mathrm{CO}_{2}$ could be driving the cometary activity, evolutionary models (Meech et al. 2013) which attempt to match the brightness measurements of C/2011 L4 are needed.

In summary, our multiwavelength observations yield the following findings: 1) very fine water ice grains in the coma of C/2011 L4; 2) no gas emission was detected; 3) $\mathrm{C} / 2011$ L4 is unusually dust-rich; 4) the dust coma seemed to becoming redder with decreasing $\mathrm{r}_{h}$.

\section{ACKNOWLEDGMENT}

We thank Jason X. Prochaska and Kate Rubin for helping us with the GMOS data reduction and Zahed Wahhaj, David Jewitt for valuable discussions and constructive suggestions. We thank the JCMT staff for their assistance. B.Y. was supported by the NASA Astrobiology Institute under Cooperative Agreement No. NNA08DA77A issued through the Office of Space Science. Work was supported in part by NSF grant AST1010059 .

\section{REFERENCES}

A'Hearn, M. F., et al. 2011, Science, 332, 1396

A'Hearn, M. F., Millis, R. C., Schleicher, D. O., Osip, D. J., \& Birch, P. V. 1995, Icarus, 118, 223

A'Hearn, M. F., Schleicher, D. G., Millis, R. L., Feldman, P. D., \& Thompson, D. T. 1984, The AJ, 89, 579

Agarwal, J., Müller, M., \& Grün, E. 2007, Space Sci. Rev., 128, 79

Baldridge, A. M., Hook, S. J., Grove, C. I., \& Rivera, G. 2009 Remote Sens. Environ., 113, 711

Bar-Nun, A., \& Laufer, D. 2003, Icarus, 161, 157

Beer, E. H., Podolak, M., \& Prialnik, D. 2006, Icarus, 180, 473

Biver, N., et al. 2002, Earth Moon and Planets, 90, 323

—. 2012, Central Bureau Electronic Telegrams, 3230, 1

Bobrovnikoff, N. T. 1954, AJ, 59, 356

Boehnhardt, H. 2004, Split comets, ed. M. C. F. H. U. Keller \& H. A. Weaver (University of Arizona Press, Tucson), 301-316

Clark, R. N., Swayze, G. A., Wise, R., Livo, K. E., Hoefen, T. M. Kokaly, R. F., \& Sutley, S. J. 2003, in American Astronomical Society, 35th DPS meeting, Vol. 35

Cochran, A. L., Barker, E. S., Ramseyer, T. F., \& Storrs, A. D. 1992, Icarus, 98, 151

Cushing, M. C., Vacca, W. D., \& Rayner, J. T. 2004, Publications of the Astronomical Society of the Pacific, 116, 362

Davies, J. K., Roush, T. L., Cruikshank, D. P., Bartholomew, M. J., Geballe, T. R., Owen, T., \& de Bergh, C. 1997, Icarus, 127,238

Enzian, A., Cabot, H., \& Klinger, J. 1997, A\&A, 319, 995

Fulle, M. 2004, Motion of cometary dust, 565-575

Hapke, B. 1993, Theory of reflectance and emittance spectroscopy

Hartmann, W. K., \& Cruikshank, D. P. 1984, Icarus, 57, 55

Hiroi, T., Zolensky, M. E., Pieters, C. M., \& Lipschutz, M. E. 1996, Meteoritics and Planetary Science, 31, 321

Hsieh, H. H., Yang, B., Haghighipour, N., et al. 2012, AJ, 143, 104

Hughes, D. W. 1990, QJRAS, 31, 69

Ivanova, O., Borysenko, S., \& Golovin, A. 2014, Icarus, 227, 202

Jewitt, D. 2009, AJ, 137, 4296

Jewitt, D. C., \& Luu, J. 2004, Nature, 432, 731

Jewitt, D., \& Matthews, H. 1999, AJ, 117, 1056

Jewitt, D., \& Meech, K. J. 1988, AJ, 96, 1723
Kawakita, H., Watanabe, J.-i., Ootsubo, T., Nakamura, R., Fuse, T., Takato, N., Sasaki, S., \& Sasaki, T. 2004, ApJ, 601, L191 Langland-Shula, L. E., \& Smith, G. H. 2011, Icarus, 213, 280 Lien, D. J. 1990, ApJ, 355, 680

Lisse, C. M., et al. 2013, Central Bureau Electronic Telegrams, 3598,2

Lisse, C. M., A'Hearn, M. F., Hauser, M. G., et al. 1998, ApJ, 496, 971

Mastrapa, R. M., Sandford, S. A., Roush, T. L., Cruikshank, D. P., \& Dalle Ore, C. M. 2009, The ApJ, 701, 1347

Meech, K. J., Yang, B., Kleyna, J., et al. 2013, ApJ, 776, L20

Meech, K. J., \& Svoren, J. 2004, Using cometary activity to trace

the physical and chemical evolution of cometary nuclei, 317-335

Niedner, M. B., Jr. 1980, ApJ, 241, 820

Notesco, G., Bar-Nun, A., \& Owen, T. 2003, Icarus, 162, 183

Prialnik, D. 1992, ApJ, 388, 196

Rayner, J. T., Toomey, D. W., Onaka, P. M., Denault, A. J., Stahlberger, W. E., Vacca, W. D., Cushing, M. C., \& Wang, S. 2003, PASP, 115, 362

Sanzovo, G. C., Singh, P. D., \& Huebner, W. F. 1996, A\&AS, 120,301

Senay, M. C., \& Jewitt, D. 1994, Nature, 371, 229

Singh, P. D., de Almeida, A. A., \& Huebner, W. F. 1992, AJ, 104, 848

Storrs, A. D., Cochran, A. L., \& Barker, E. S. 1992, Icarus, 98, 163

Trujillo, C. A., Brown, M. E., Barkume, K. M., Schaller, E. L., \& Rabinowitz, D. L. 2007, ApJ, 655, 1172

Wainscoat, R., et al. 2011, IAUC, 9215

Weiler, M., Rauer, H., Knollenberg, J., Jorda, L., \& Helbert, J. 2003, A\&A, 403, 313

Williams, G. V. 2013, Minor Planet Electronic Circulars, 2013-V48

Whipple, F. L. 1978, Nature, 273, 134

Yang, B., Jewitt, D., \& Bus, S. J. 2009, AJ, 137, 4538

Yang, B., \& Sarid, G. 2010, IAUC, 9139 
TABLE 1

Observational Parameters for C/2011 L4 (Panstarrs)

\begin{tabular}{|c|c|c|c|c|c|c|}
\hline UT Date & $\begin{array}{l}r_{h}{ }^{\mathrm{a}} \\
(\mathrm{AU})\end{array}$ & $\begin{array}{c}\Delta \mathrm{b} \\
(\mathrm{AU})\end{array}$ & $\begin{array}{l}\alpha^{\mathrm{c}} \\
\operatorname{deg}\end{array}$ & Tel. & Inst. & $\begin{array}{l}\lambda \mathrm{d} \\
\mu \mathrm{m}\end{array}$ \\
\hline 2012 Mar. 18 & 5.248 & 4.956 & 10.71 & IRTF & SpeX & $0.80-2.5$ \\
\hline 2012 Apr. 22 & 4.882 & 4.081 & 7.80 & Gemini & GMOS & $0.38-0.6$ \\
\hline 2012 May 15 & 4.635 & 3.650 & 3.21 & Gemini & GMOS & $0.38-0.6$ \\
\hline 2012 Jun. 06 & 4.392 & 3.400 & 3.13 & Gemini & GNIRS & $0.85-2.5$ \\
\hline 2012 July. 12 & 3.980 & 3.328 & 12.30 & Gemini & GMOS & $0.38-0.6$ \\
\hline 2012 July. 19 & 3.898 & 3.350 & 13.61 & IRTF & SpeX & $0.80-2.5$ \\
\hline 2012 Aug. 25-27 & 3.437 & 3.526 & 16.63 & JCMT & HARP & $800-922$ \\
\hline 2012 Oct. $20-22$ & 2.713 & 3.520 & 10.49 & JCMT & HARP & $800-922$ \\
\hline 2012 Nov. $19-21$ & 2.310 & 3.256 & 5.62 & JCMT & HARP & $800-922$ \\
\hline
\end{tabular}

a Heliocentric distance

b Geocentric distance.

c Solar phase angle (Sun-object-Earth)

$\mathrm{d}$ Wavelength coverage.

TABLE 2

Af $\rho$ AND DUST PRoduction RATES FOr C/2011 L4 (PANSTARrs)

\begin{tabular}{cccccccc}
\hline \hline UT Date & $\begin{array}{c}f_{C N^{\mathrm{a}}} \\
\left(\mathrm{erg} / \mathrm{cm}^{2} / \mathrm{s} / \AA\right)\end{array}$ & $\begin{array}{c}\mathrm{Q}_{C N^{\mathrm{b}}} \\
(\mathrm{mol} / \mathrm{s})\end{array}$ & $\begin{array}{c}\mathrm{Q}_{\mathrm{H}_{2} \mathrm{O}^{\mathrm{c}}} \\
(\mathrm{mol} / \mathrm{s})\end{array}$ & $\begin{array}{c}\mathrm{Af} \rho \\
(\mathrm{m})\end{array}$ & $\begin{array}{c}\dot{M}_{d}{ }^{\mathrm{d}} \\
(\mathrm{kg} / \mathrm{s})\end{array}$ & $\begin{array}{c}\dot{M}_{g}{ }^{\mathrm{e}} \\
(\mathrm{kg} / \mathrm{s})\end{array}$ & $\chi^{\mathrm{f}}$ \\
\hline 2012 Apr. 22 & $1.51 \times 10^{-15}$ & $<1.68 \times 10^{25}$ & $<5.9 \times 10^{27}$ & $110.2 \pm 8.0$ & 670 & $<200$ & $>3.4$ \\
2012 May 15 & $1.92 \times 10^{-15}$ & $<1.78 \times 10^{25}$ & $<6.2 \times 10^{27}$ & $116.9 \pm 8.0$ & 650 & $<212$ & $>3.1$ \\
2012 July. 12 & $1.90 \times 10^{-15}$ & $<1.41 \times 10^{25}$ & $<5.0 \times 10^{27}$ & $128.9 \pm 9.0$ & 730 & $<168$ & $>4.3$ \\
\hline
\end{tabular}

${ }^{a} 3 \sigma$ upper limit of the $\mathrm{CN}$ band flux

b $\mathrm{CN}$ production rate, upper limit

c $\mathrm{H}_{2} \mathrm{O}$ production rate, upper limit, assuming $\mathrm{Q}_{C N} / \mathrm{Q}_{\mathrm{H}_{2} \mathrm{O}} \sim 350$ (A'Hearn et al. 1995)

d Dust mass loss rate, assuming a size distribution of $\mathrm{f}(\mathrm{a}) \sim \mathrm{a}^{-4}$

e Gas mass loss rate, assuming $\dot{M}_{g}=3.4 \times 10^{-26} \mathrm{Q}_{H_{2} \mathrm{O}}$ (Sanzovo et al. [1996).

f Dust-to-gas ratio. 


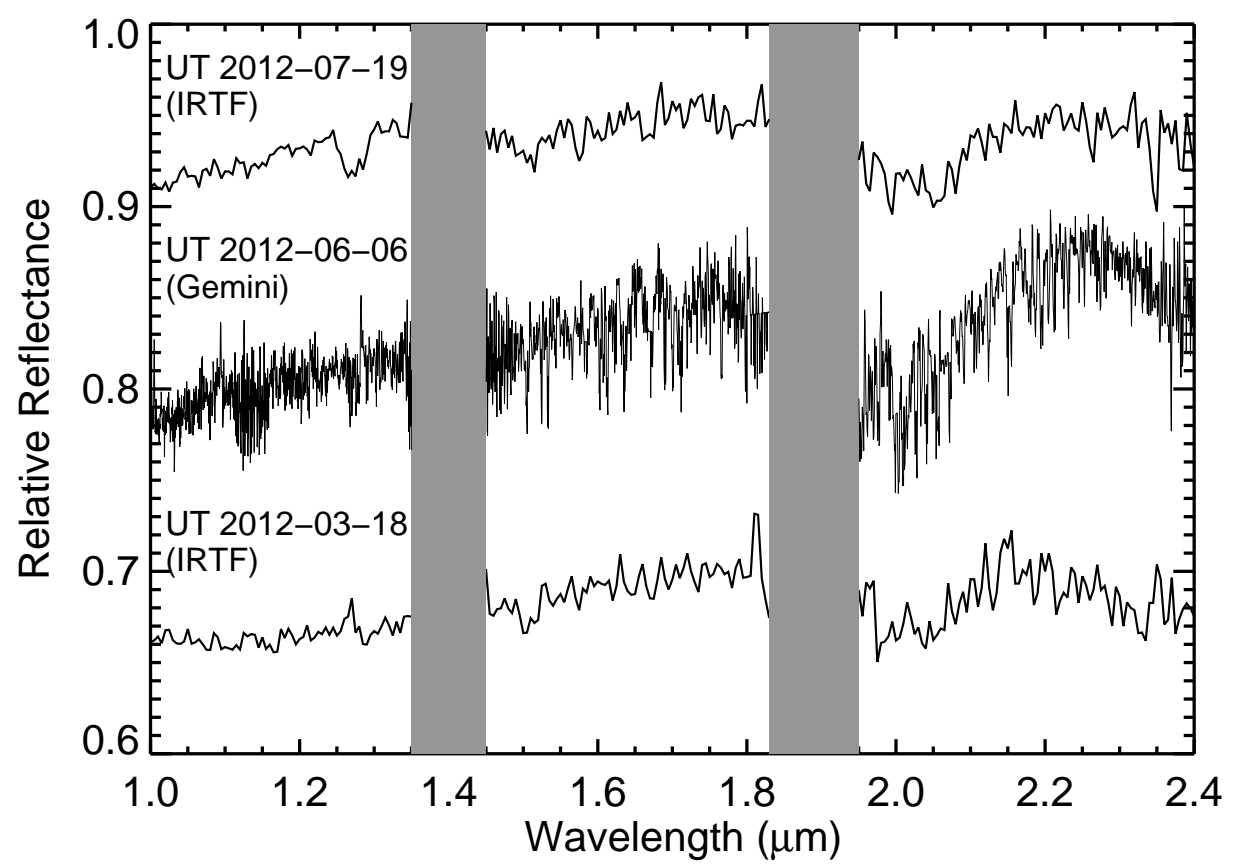

FIG. 1. - NIR spectra of C/2011 L4, taken with the Gemini-N and IRTF telescopes. All the spectra consistently show reddish continuum and strong absorption feature centered at $2.0 \mu \mathrm{m}$. The Gemini spectrum appears noisier than the IRTF spectra because the former has a higher spectral resolution $\left(\mathrm{R}_{G} \sim 560\right)$ than the latter $\left(\mathrm{R}_{I} \sim 150\right)$. Spectral regions that are contaminated by atmospheric absorptions are blocked by grey bars. In the March data (bottom panel), the emission-like feature at 1.82 and $2.15 \mu \mathrm{m}$ in the March data are artifacts due to telluric absorption. 

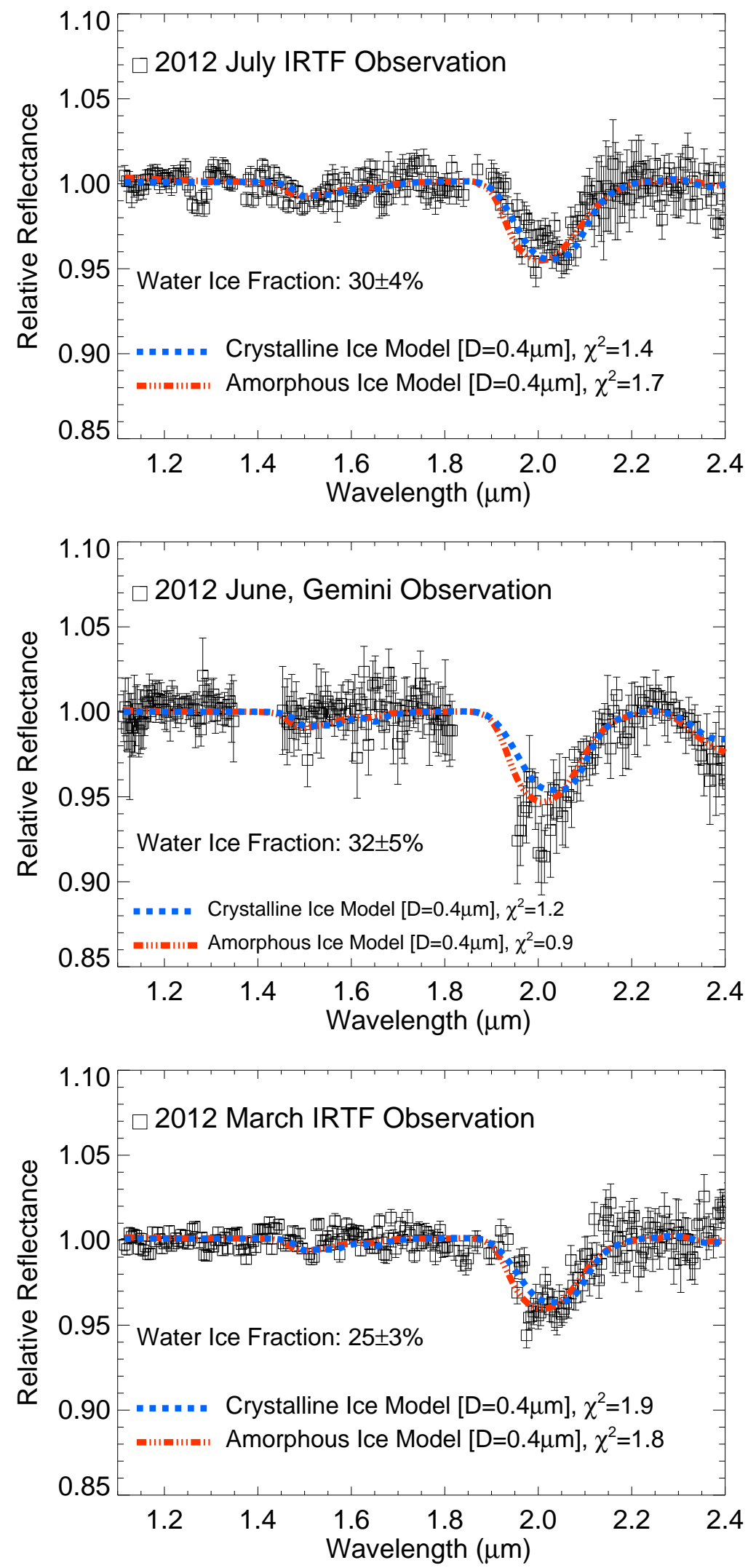

FIG. 2.- The open squares are the continuum-removed NIR spectra of C/2011 L4. The red and the blue dashed lines are simple spectral models using pure amorphous water ice and crystalline ice mixed with spectrally neutral material. The model is fitted by minimizing chi-square with an iterative procedure. The chi-square values are calculated from 1.95 to $2.2 \mu \mathrm{m}$, focusing on the fit to the $2.0 \mu \mathrm{m}$ band. The discrepancy seen at $2.0 \mu \mathrm{m}$ is due to imperfect removal of a $\mathrm{CO}_{2}$ absorption band due to Earth's atmosphere. 

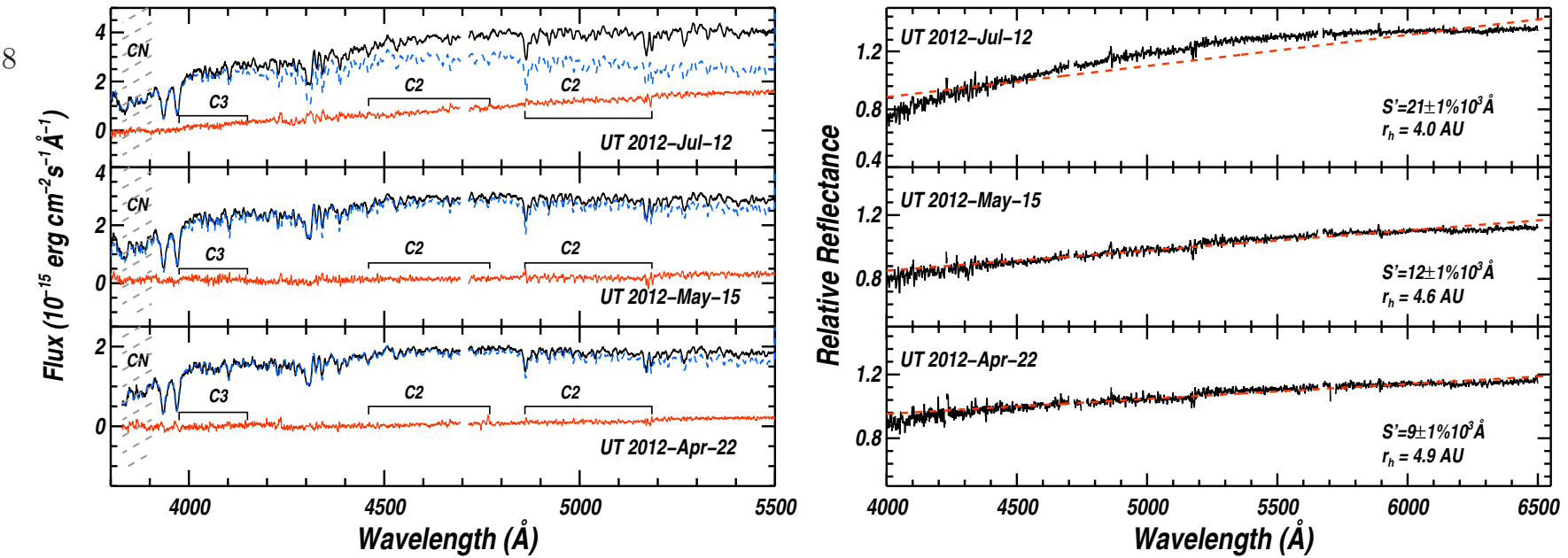

FIG. 3.- (a) Flux calibrated optical spectra of C/2011 L4 are shown in black. Blue lines are the scaled solar analog spectra and red lines are residuals after removing the solar analog continuum. The expected $\mathrm{CN}, \mathrm{C}_{3}$ and $\mathrm{C}_{2}$ bands are marked, the bandpasses are taken from (Cochran et al. 1992). Small emission-like features in the residual spectra are artifacts due to imperfect removal of the telluric absorptions. (b) Reflectance spectra of C/2011 L4 (black) and linear regression fits (red). The spectral slope seems to be correlated with the heliocentric distance, i.e. the comet spectrum becomes redder as the heliocentric distance decreases. Two gaps at $4700 \AA$ and $5700 \AA$ are caused by $\sim$ $2.8^{\prime \prime}$ gaps between the detector chips of GMOS. 